\title{
Maternal Aggression Is Reduced in Neuronal Nitric Oxide Synthase-Deficient Mice
}

\author{
Stephen C. Gammie and Randy J. Nelson \\ Departments of Psychology and Neuroscience, Behavioral Neuroendocrinology Group, The Johns Hopkins University, \\ Baltimore, MD 21218
}

Lactating females express rapid extremes in behavior, ranging from gentle nurturance toward offspring to fiercely protective aggression against intruders. Although males often behave aggressively against intruders, female rodents usually express aggression only when rearing and protecting pups. Nitric oxide (NO) inhibits male aggression; however, its role in maternal aggression is unknown. In the present study, female mice with targeted disruption of the neuronal nitric oxide synthase gene (nNOS-/-) displayed significant deficits in maternal aggression relative to wild-type (WT) mice in terms of percentage displaying aggression, the average number of attacks against a male intruder, and the total time spent attacking the male intruder. The nNOS-/- mice displayed normal pup retrieval behavior. Because the specific deficits in maternal aggression in the $\mathrm{nNOS}-1-$ mice suggested a possible role for $\mathrm{NO}$ in maternal aggression, we combined behavioral testing of WT mice with immunohistochemistry for citrulline, an indirect marker of NO synthesis, to examine indirectly NO synthesis during maternal aggression. A significant increase in the number of citrulline-positive cells was identified in the medial preoptic nucleus, the suprachiasmatic nucleus, and the subparaventricular zone regions of the hypothalamus in aggressive lactating females relative to control mice. In other regions of the brain, no changes in the number of citrulline-positive cells were observed across either groups or treatments. These results provide two indirect lines of evidence that NO release is associated with maternal aggression.

Key words: nitric oxide; neuronal nitric oxide synthase; maternal aggression; citrulline; hypothalamus; mice
Female mice exhibit fierce aggression toward intruders when they are lactating and rearing pups (Svare, 1990). Because this temporal expression of aggression, termed maternal aggression, is highly conserved among mammals, it has been hypothesized that this behavior increases the likelihood of survival of the offspring (Wolff, 1985). Female mice can also show a type of territorial aggression toward other females, but this aggression is much less fierce than that shown toward males during maternal aggression and is thought to be neurally similar to male aggression (Parmigiani et al., 1998). In support of this idea, low levels of a serotonin agonist can eliminate female-female aggression and male aggression but have no effect on maternal aggression (Parmigiani et al., 1998). Because females only express maternal aggression in association with the rearing of pups, it is reasonable that females developed a specialized mechanism for the control of maternal aggression that differs from other forms of aggression.

Maternal aggression, along with other specific maternally related behaviors, such as nursing and pup retrieval, constitute maternal behavior. The complex mechanisms controlling maternal aggression and other maternal behaviors are best understood in rats. Although steroid hormones, such as estradiol and proges-

\footnotetext{
Received April 22, 1999; revised June 22, 1999; accepted June 28, 1999.

This work was supported by National Institutes of Health Grants MH 57535 and MH 57760 to R.J.N and National Institutes of Health National Research Service Award MH 12371-01 to S.C.G. We thank Drs. M. J. L. Eliasson and S. H. Snyder for use of the anti-citrulline antibodies and Drs. T. M. Dawson and V. L. Dawson for access to the nNOS $-/-$ mice. We also thank L. J. Kriegsfeld, B. D. Spar, and C. Y. Wan for technical assistance and Drs. M. Gallagher and G. F. Ball for providing criticism of this manuscript.

Correspondence should be addressed to Stephen C. Gammie, 3400 N. Charles Street, Room 225, Ames Hall, Department of Psychology, Behavioral Neuroendocrinology Group, The Johns Hopkins University, Baltimore, MD 21218.

Copyright (C) 1999 Society for Neuroscience 0270-6474/99/198027-09\$05.00/0
}

terone, released during pregnancy enable female rats to express maternal behaviors, including aggression (Mann et al., 1984; Stern and McDonald, 1989; Bridges, 1996), there is no conclusive evidence that these steroids are necessary for the expression of maternal aggression once a dam is lactating. In rats, the sensory input produced by the suckling of pups plays a role in activating and maintaining maternal aggression (Stern and Kolunie, 1993), and this action may result from suckling-induced increases in central serotonin production and release (Kordon et al., 1973). Also in rats, the neuropeptide oxytocin may act centrally to facilitate maternal aggression (Giovenardi et al., 1998). Less is known about the possible neural basis of maternal aggression in mice, but recent work indicates that the biogenic amine norepinephrine triggers the onset of many murine maternal behaviors, such as pup retrieval, around the time of parturition (Thomas and Palmiter, 1997).

In contrast to females, male mice show high levels of aggression toward intruders throughout the year. In many rodents, the neuropeptide vasopressin (AVP) has an excitatory effect on male aggression (Ferris et al., 1997), whereas serotonin has an inhibitory action (Olivier et al., 1995). Specific deletion of the neuronal nitric oxide synthase (nNOS) gene (nNOS-/-) and the pharmacological inhibition of nNOS both result in increased aggression in male mice (Nelson et al., 1995; Demas et al., 1997). These results suggest that the gas nitric oxide (NO), which can act either anterogradely or retrogradely as a neuromodulator within the CNS (Bredt and Snyder, 1992), has an inhibitory action on male aggression. The normally quiescent behavior of virgin females was not altered in nNOS-/- mice (Nelson et al., 1995), but an examination of maternal aggression was not previously conducted. Although NO has been linked to the timing of parturition 
in rats (Okere et al., 1996) and to the formation of olfactory memories in lactating sheep (Kendrick et al., 1997), to date, no work has examined the relationship of NO to maternal aggression. In this study, we sought first to examine how the targeted disruption of the nNOS gene affected the production of maternal aggression in $\mathrm{nNOS}-1-$ mice. We then went on to explore indirectly the dynamics of NO synthesis during maternal aggression by combining behavioral testing with immunohistochemistry for citrulline, an indirect marker for NO synthesis.

\section{MATERIALS AND METHODS}

Behavioral testing. Thirteen wild-type (WT) female house mice (Mus musculus) of the C57B6J strain and 9 nNOS-/- (Huang et al., 1993) female mice were paired with males. After impregnation, the females were housed individually in polypropylene cages in a colony room with a 16/8 light/dark schedule. The date of birth was considered postpartum day 0 , and litters were culled to six pups to decrease variability in maternal aggression (Maestripieri, 1990). Beginning on day 4 and continuing for each day until day 10, each female was exposed to an intruder male for $10 \mathrm{~min}$ between 8:00 A.M. and 12:00 P.M. The pups were removed from the cage $3 \mathrm{~min}$ before the behavioral test, and each test session with a male was recorded on videotape and subsequently analyzed off-line to quantify aggressive behaviors by the female. Removal of the pups from a mother just before an aggressive test does not diminish the expression of maternal aggression (Svare et al., 1981). The intruder males were sexually naive and group housed and were either of the CD1 or C57B6J strain. The levels of maternal aggression elicited by the two strains were identical (data not shown). After each test, the pups were weighed and randomly distributed throughout the home cage. The time to retrieve the first and fourth pup was recorded for each session. For a given animal, the data from the day that the animal showed peak aggression were used for group comparison and statistical analysis.

Citrulline immunocytochemistry. Seven lactating WT females (between postpartum days 8 and 10), five lactating $n N O S-/-$ females, five WT virgin females, and five WT males (all individually housed) were exposed to a sexually naive intruder (group-housed) male for $10 \mathrm{~min}$. An additional five WT lactating females were exposed to an intruder male for 10 min that had previously been anesthetized with sodium pentobarbital and was immobile. Immediately after a behavioral test, each test animal was briefly anesthetized for 1 min with methoxyflurane vapor (Mallinckrodt Veterinary, Inc., Mundelein, IL) and further anesthetized with an overdose of sodium pentobarbital. Animals were perfused through the heart with an oxygenated Krebs'-Heinzleit buffer (in mM: $118 \mathrm{NaCl}, 4.7$ $\mathrm{KCl}, 2 \mathrm{CaCl}_{2}, 1.2 \mathrm{MgSO}_{4}, 1.2 \mathrm{KH}_{2} \mathrm{PO}_{4}, 25 \mathrm{NaHCO}_{3}$, and 11 glucose, $\mathrm{pH}$ 7.4), followed by a $5 \%$ glutaraldehyde $-0.5 \%$ formaldehyde solution containing $0.2 \% \mathrm{Na}_{2} \mathrm{~S}_{2} \mathrm{O}_{5}$ in $0.1 \mathrm{M}$ PBS (Eliasson et al., 1997). Five WT lactating females, six WT virgin females, and nine WT males were also perfused as described, but no behavioral test was conducted before fixation. All tests and perfusions were performed between 8:00 A.M. and 12:00 P.M. After the perfusions, the brains were removed, post-fixed overnight at $4^{\circ} \mathrm{C}$, and placed in a $20 \%$ glycerol cryoprotectant for $2 \mathrm{~d}$. The brains were frozen on dry ice immediately before sectioning at 40 $\mu \mathrm{m}$ on a cryostat. The brain sections were collected in PBS and reduced for 30 min with $0.5 \% \mathrm{NaBH}_{4}$ and $0.2 \% \mathrm{Na}_{2} \mathrm{~S}_{2} \mathrm{O}_{5}$ in $10 \mathrm{~mm}$ PBS with 0.19 $\mathrm{mm} \mathrm{NaCl}, \mathrm{pH} 7.4$. Subsequently, the sections were washed in PBS in the presence of $0.2 \%$ Triton X-100 (PBS-X), blocked in 5\% normal goat serum for $1 \mathrm{hr}$, and incubated for $2 \mathrm{~d}$ at $4^{\circ} \mathrm{C}$ with rabbit anti-citrulline antibodies $(1: 10,000)$ that had been preabsorbed against arginine (Pasqualotto et al., 1991; Eliasson et al., 1997). After washes in PBS-X, the sections were incubated overnight at $4^{\circ} \mathrm{C}$ in biotinylated goat anti-rabbit secondary antibodies (1:1000), washed in PBS-X, exposed to an avidinbiotin complex (Vector Laboratories, Burlingame, CA) for $1 \mathrm{hr}$, washed again in PBS-X, and visualized using diaminobenzidine as a chromagen. The sections were mounted and counterstained with thionin before coverslips were applied.

Cell counting and statistical analysis. From each animal, brain sections corresponding to bregma -0.34 , bregma -0.46 , and bregma $-0.70 \mathrm{~mm}$ (Franklin and Paxinos, 1997) were identified, and cells with citrulline immunoreactivity (IR) in the medial preoptic area (MPOA), the suprachiasmatic nucleus (SCN), and the subparaventricular zone (SPa) regions of the hypothalamus were counted in one hemisegment. The SCN resides in all three sections, but the MPOA occurs only at bregma -0.34 and $-0.46 \mathrm{~mm}$ and lies just dorsal to the SCN, whereas the SPa occurs only at bregma $-0.70 \mathrm{~mm}$ (Franklin and Paxinos, 1997) and also lies just dorsal to the SCN. The control region for examining overall citrulline-IR was a square region of $500 \times 500 \mu \mathrm{m}$ placed in the caudate putamen at the level of bregma $0.14 \mathrm{~mm}$. The boxed area used for counting cells was placed at the lateral most edge of the caudate putamen and at the same ventral level as the bottom of the lateral ventricles. All cell counting was performed by eye at $400 \times$ magnification under a microscope. The counting of cells with citrulline-IR was performed three times independently, and less than a $5 \%$ variation occurred between counts. For statistical analysis across groups and treatments, a two-way ANOVA was used. An unpaired Student's $t$ test was used only for comparisons of lactating females exposed to an active male intruder with lactating females exposed to an anesthetized intruder. Stereology was not used during cell counting because the distance between the representative sections was greater than $100 \mu \mathrm{m}$, a size greater than the average size of cell bodies in these regions of the brain.

\section{RESULTS \\ Maternal behavior and aggression of lactating wild- type and nNOS-/- mice}

We examined the levels of maternal aggression in lactating mice that were lacking the second exon of the nNOS gene (nNOS-/-) and showing $>95 \%$ loss of NO production in the CNS (Huang et al., 1993; Eliasson et al., 1997). WT C57B6J strain mice were used as controls because these mice share $>99.9 \%$ genetic similarity with the transgenic mice. For the maternal aggression tests, lactating females were individually housed with their pups, and the pups were removed 3 min before the introduction of a sexually naive male intruder in the home cage. Each aggression test lasted $10 \mathrm{~min}$. Compared with lactating WT dams, lactating nNOS $-/-$ mice display significant deficits in the production of maternal aggression in terms of percentage displaying aggression, the average number of attacks against a male intruder during the 10 min test, and the total time spent attacking the male intruder (Fig. $1 A-C$ ). The greatest behavioral deficit in the nNOS-/females was the $\sim 90 \%$ decrease in the amount of time spent actively attacking or biting the intruder male (Fig. 1C). Because the C57B6J strain is known to show lower levels of maternal aggression relative to other mice strains (Svare, 1990), aggression was tested for 7 consecutive days, beginning on day 4 of parturition and ending on day 10. Despite this long investigation time, the nNOS $-/-$ mice still displayed extremely low levels of maternal aggression. A qualitative difference in maternal aggression was also apparent because, in the rare instances when the nNOS $-/-$ females did attack the intruder males, the bites were best described as grabs, and usually the intruder males did not respond. In contrast, when the WT females attacked the males, the males usually fled or became engaged in a fight. One behavioral feature that did not differ between the nNOS $-/-$ and WT mice was the amount of time spent sniffing the intruder male (Fig. 1D)

In contrast to the deficits in maternal aggression, the pup retrieval behavior was almost identical for the WT and nNOS-/- mice (Fig. 2A). Unexpectedly, the pups of the nNOS $-/-$ females displayed significant increases in terms of average weight compared with WT pups (Fig. 2B). Whether the elevated pup weight resulted from the behavior, or physiology, of the pups, the mothers, or both, was not determined.

\section{Pattern of citrulline immunoreactivity in the brain of aggressive and nonaggressive mice}

The specific deficits in maternal aggression in the nNOS $-/-$ mice suggested that NO might normally be involved in the production of maternal aggression in WT mice. To determine, indirectly, whether NO release might be associated with maternal aggres- 

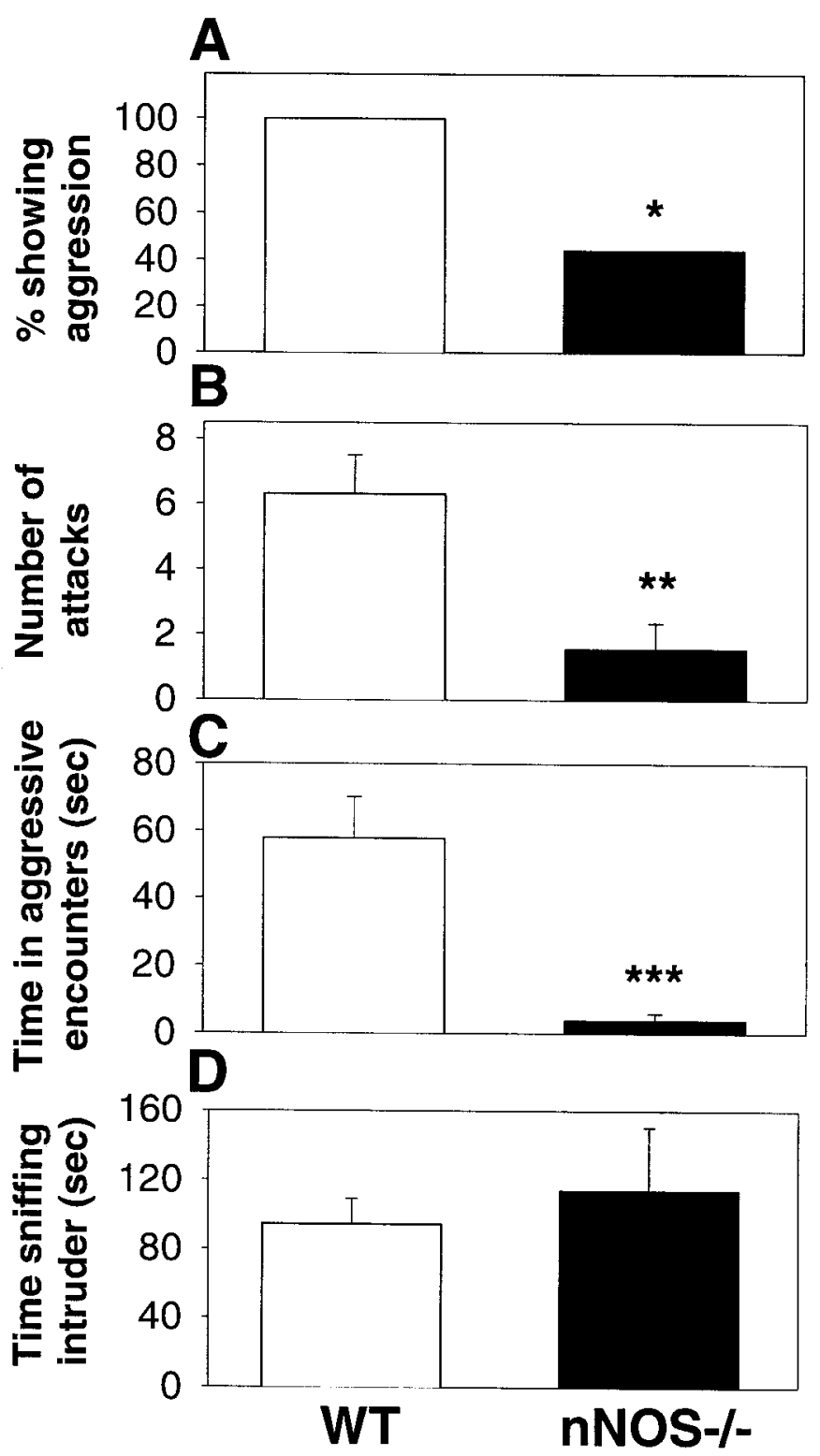

Figure 1. Specific deficits in maternal aggression in lactating nNOS-/mice. Using a resident-intruder test paradigm, nNOS $-/-$ lactating females showed an impaired ability to express maternal aggression toward an intruder male in terms of the percentage of females showing any aggression $(A)$, the average number of attacks during the 10 min test period $(B)$, and the average amount of time spent engaged in an agonistic encounter $(C)$. No differences were observed in the amount of time spent sniffing the intruder $(D)$. Error bars represent means \pm SE. $* p<0.05$; ${ }^{* *} p<0.01 ;{ }^{* *} p<0.001$; one-way ANOVA on ranks for $A$; unpaired Student's $t$ test for $B-D$.

sion, we combined aggressive behavioral testing with immunohistochemistry for citrulline, an indirect marker of NO release. Citrulline is produced within a cell when NO is enzymatically cleaved from the precursor arginine, and, unlike NO, which readily diff uses out of the cell and has a half-life on the order of seconds, citrulline remains trapped within the cell and can be detected for longer periods of time (Eliasson et al., 1997; Moroz et al., 1999). In earlier histochemical studies in the mouse brain, citrulline-IR was always found to colocalize with cells containing NOS, indicating that it is a reflection of NO activity and not urea
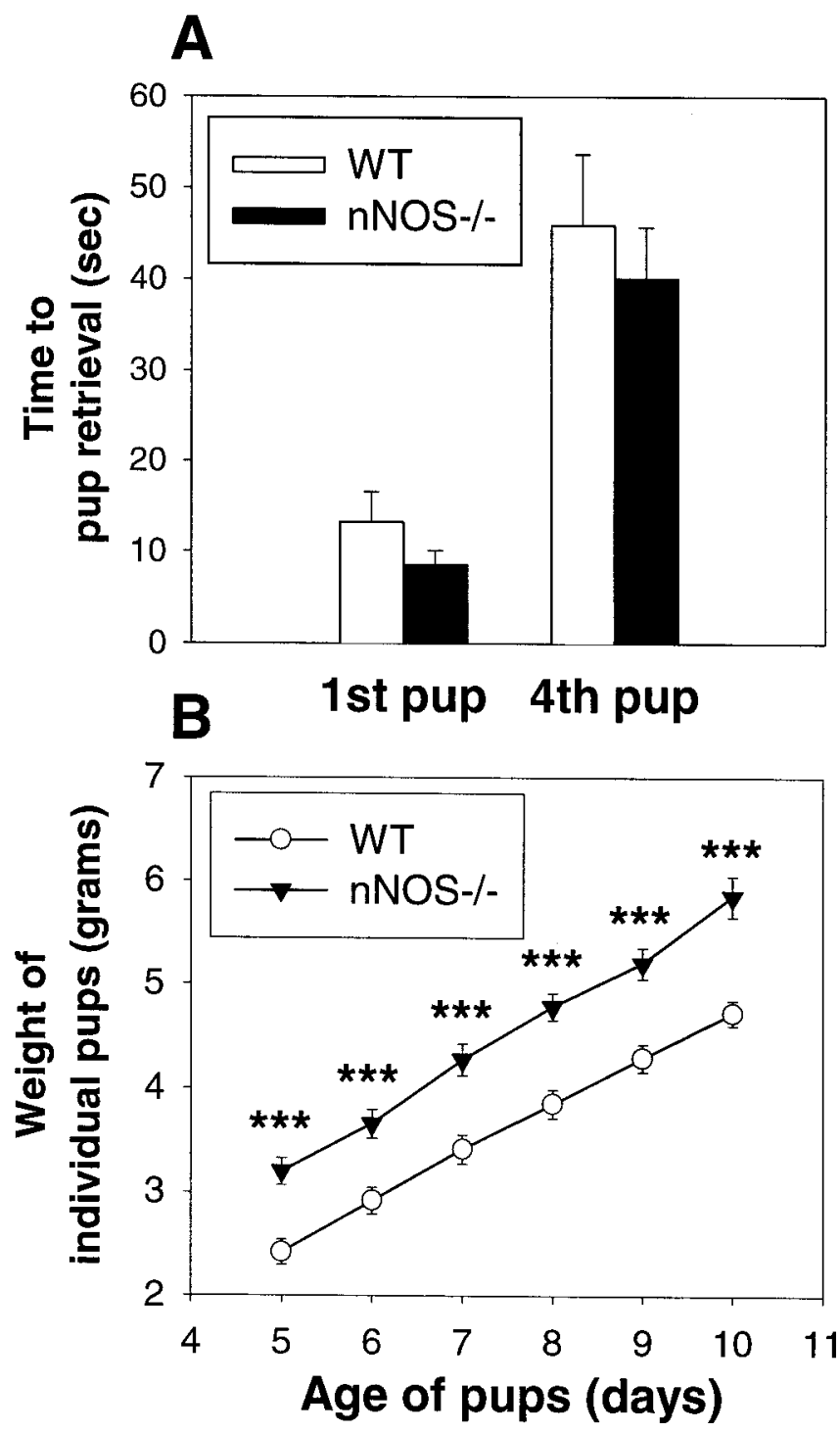

Figure 2. nNOS-/- lactating females exhibit normal nurturing abilities. $A$, The average time spent retrieving the first and fourth pup in a pup retrieval test is almost identical between nNOS $-/-$ and WT lactating females $(p>0.05)$. Error bars represent means \pm SE. $B$, The average weight of individual pups of the nNOS $-/-$ females is significantly greater than for the pups of the WT females. ${ }^{* *} p<0.001$; unpaired Student's $t$ test.

cycle activity (Eliasson et al., 1997). In these studies, we first examined the levels of citrulline-IR in the brains of WT lactating female mice immediately after an aggressive encounter with an intruder male, using the same behavioral testing paradigm used previously to determine levels of maternal aggression, and compared those with levels in an unstimulated lactating female control. To help control for the activation of neural circuits resulting from the stimulation of visual and olfactory pathways in the female by the intruder male, we also exposed lactating females to immobile intruder males anesthetized with sodium pentobarbital and examined the brains of the females for citrulline-IR after the behavioral test. In this latter case, the females were never aggressive toward the immobile intruder but did spent large amounts of time sniffing and examining the intruder.

As seen in Figure 3, when a WT female was exposed to an 
Figure 3. Citrulline-IR is significantly altered in the hypothalamus in lactating, aggressive female mice compared with control, nonaggressive lactating females. Representative photomicrographs showing citrulline-IR in the hypothalamus of a lactating female exposed to an immobile, anesthetized male intruder for 10 min that did not elicit an aggressive response $(A, C, E)$ and a lactating female exposed to an active male intruder that triggered an aggressive response $(B, D$, $F$ ). The paired sections in $A$ and $B, C$ and $D$, and $E$ and $F$ correspond to bregma -0.34 , bregma -0.46 , and bregma -0.70 mm (Franklin and Paxinos, 1997), respectively. The MPOA, SCN, and SPa regions are indicated on the photomicrographs. Higher power representative photomicrographs showing different levels of citrulline-IR in the neuronal processes in the hypothalamus of unstimulated lactating females $(G)$ and lactating females exposed to a male intruder for $10 \min (H)$. The brain regions in $G$ and $H$ correspond to bregma $-0.46 \mathrm{~mm}$ and include the SCN and MPOA. Arrowheads indicate cells with citrulline-IR in the cell bodies only, and arrows correspond to cells with citrulline-IR within neuronal processes. Scale bars, $100 \mu \mathrm{m}$.
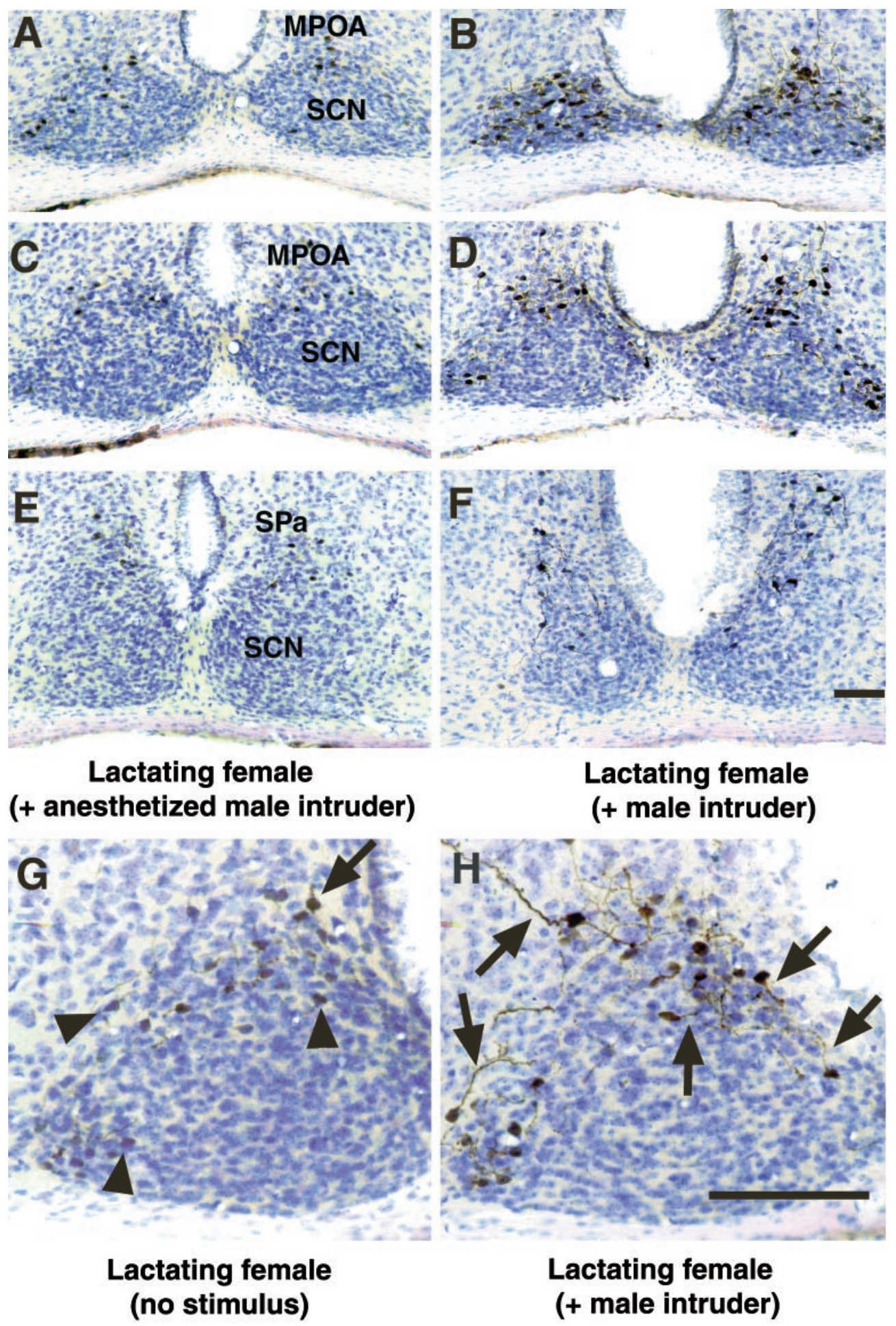

\section{Lactating female (+ male intruder)}

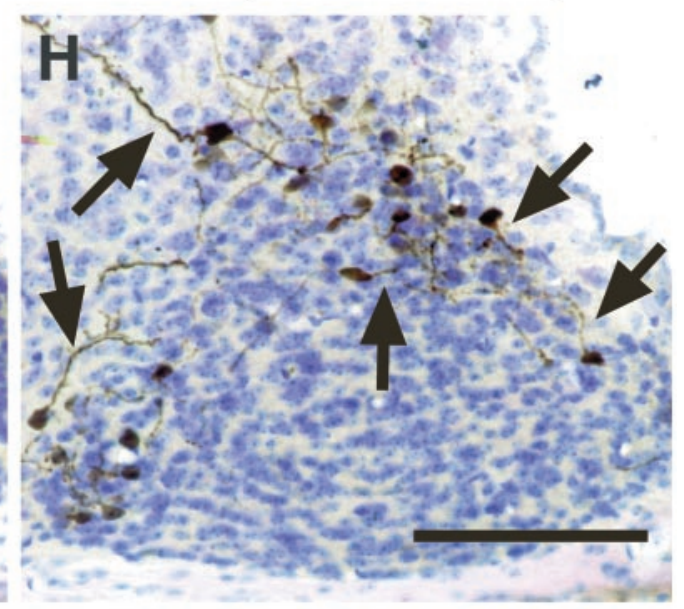

Lactating female
(+ male intruder) active intruder male and produced aggression, an increase in the number of cells exhibiting citrulline-IR occurs within subregions of the hypothalamus relative to mice in the two control groups. The regions of the hypothalamus exhibiting the greatest increases in citrulline-IR in association with aggression include the MPOA, the SCN, and the SPa (Fig. 3). In addition to the increase in the number of neurons exhibiting citrulline-IR, aggressive lactating females also exhibited increases in the number of cells with detectable levels of citrulline-IR within their neuronal processes (Fig. 3G,H). Because some of the citrulline-positive cells in the
MPOA and SPa regions were closely adjoined to citrullinepositive cells in the dorsal SCN (in what is sometimes called the peri-SCN region), it was sometimes unclear to which region of the hypothalamus a particular cell should be assigned. Consequently, the combined total number of citrulline-positive cells in the MPOA, SCN, and SPa regions was used for most analyses. Cells were counted from one hemisegment of each of three sections shown (Fig. $3 A, C, E$ ) for each animal. The means from each group and treatment are shown in Figure 4, and a list of the distribution of the number of citrulline-positive cells in the dif- 


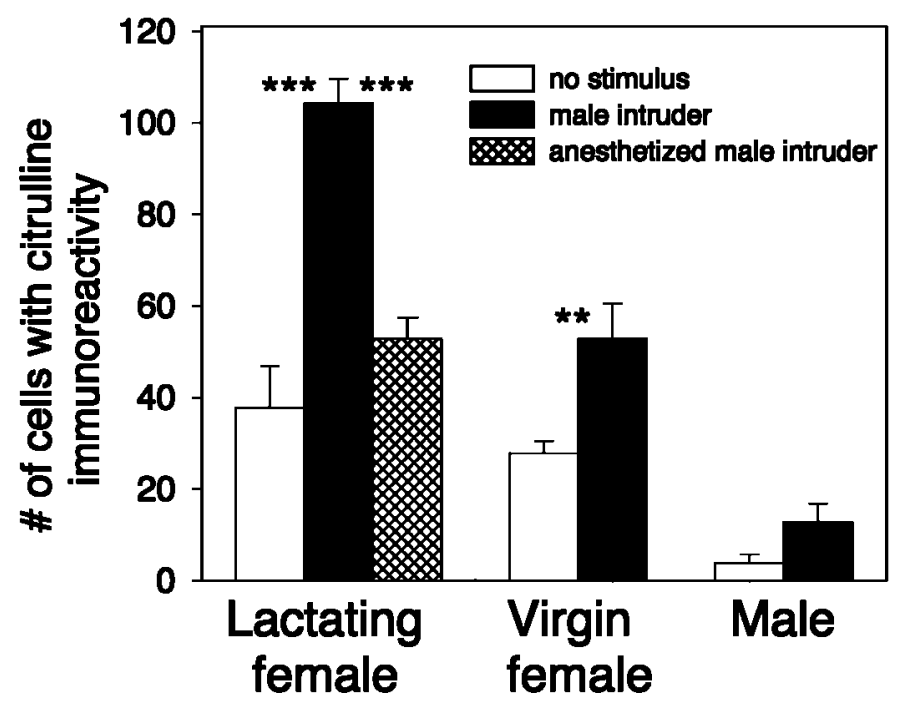

Figure 4. The average number of citrulline-positive cells in the hypothalamus differs across groups and treatments. Error bars represent means \pm SE. Cells were counted in one hemisegment of each of the three representative sections shown in Figure $3(A, C, E)$ for each animal in each group and treatment in the MPOA, SCN, and SPa subregions of the hypothalamus. Between the groups with the no stimulus treatment, the lactating females and virgin females did not differ from one another significantly, but both differed significantly from the male group $(p<$ 0.01 ). Between the groups with the active male intruder treatment, all three groups differed significantly from one another $(p<0.001)$. Within each group, statistically significant differences are shown: ${ }^{* *} p<0.01$; $* * * p<0.001$.

ferent subregions of the hypothalamus that were examined is provided in Table 1. Aggressive lactating females significantly increased the number of citrulline-positive cells relative to the two control groups in terms of total cells and cells within each of the three examined subdivisions of the hypothalamus (Fig. 4, Table 1).

To examine further whether the numbers of citrulline-positive cells increased with the levels of maternal aggression, we examined the correlation coefficient of time spent attacking the intruder and total number of citrulline-positive cells in the MPOA, $\mathrm{SCN}$, and SPa regions. We found a significant positive correlation between these two variables and a correlation coefficient of 0.816 ( $p<0.05$; Pearson product moment correlation; $n=6)$.

To examine whether the increase in number of citrulline-IR cells in lactating females exposed to an active male was specific to that life-history stage of the female, we exposed virgin females to an active male intruder for $10 \mathrm{~min}$ using the resident-intruder test and then examined the brains of the virgin females for citrullineIR. We also examined citrulline-IR in unstimulated virgin female controls. Behaviorally, the virgin females exposed to the intruder males were never aggressive. Unstimulated virgin females exhibited a lower number of citrulline-positive cells in the hypothalamus than unstimulated lactating females (Figs. 4, 5A). When virgin females were exposed to a male intruder, the number of citrulline-positive cells almost doubled relative to control (Figs. 4, $5 A, B)$, indicating that the presence of a male can stimulate increases in citrulline-IR in the absence of aggression. Still, the heightened numbers of citrulline-positive cells in the stimulated virgins were about equivalent to the two control groups of lactating females and were significantly less than the numbers observed in the aggressive lactating females (Fig. 4), suggesting that the life-history stage of the female may affect the ability to increase citrulline production in response to a male.

Isolated male mice are reliably aggressive toward intruder males. To determine whether aggressive males also showed elevations in citrulline-IR in the hypothalamus compared with unstimulated male controls, we examined the brains of males for citrulline-IR after resident-intruder aggressive testing. In five of five experiments, the resident male attacked the intruder. As seen in Figure 4, for unstimulated males, the number of citrullinepositive cells was significantly lower than for unstimulated virgin or lactating females. Additionally, after a $10 \mathrm{~min}$ aggressive test, the males showed only a slight increase in the number of citrulline-positive cells, and these levels were significantly lower than for any of the female groups. This latter result suggests that, in regard to citrulline-IR in the hypothalamus, males and females show sexually dimorphic patterns of citrulline synthesis.

nNOS $-/-$ mice lack the second exon of the nNOS gene and, in some nonhypothalamic regions of the brain, neuronal NOS activity can be detected in the nNOS-/- mice (Eliasson et al., 1997). To examine, indirectly, whether NO was being produced in the hypothalamus of the nNOS-/- female mice, we exposed three lactating $\mathrm{nNOS}-/-$ females to a male intruder for $10 \mathrm{~min}$ and examined the brains for citrulline-IR. Behaviorally, none of the nNOS - / - expressed aggression and in each case no citrulline-IR was observable in the hypothalamus (Fig. $5 D$ ).

In contrast to the changes in the hypothalamus, the number of cells with citrulline-IR was unaltered in other regions of the brain across groups and treatments. An example of citrulline-IR in the caudate putamen region of the brain in an aggressive and control lactating female is shown in Figure $6 A, B$. The mean number of citrulline-positive cells in this region did not differ significantly across groups or treatment (Fig. 6C).

\section{DISCUSSION}

\section{Maternal aggression deficits in nNOS-/- mice}

The nNOS $-/-$ mice are $>99.9 \%$ genetically identical to the C57B6J WT mice but exhibited a specific deficit in maternal aggression relative to WT. Because the C57B6J strain shows decreased maternal aggression relative to other strains, we tested the WT and nNOS-/- lactating females for maternal aggression each day for 7 consecutive days to maximize the observation of aggression. Using this paradigm, a dramatic decrease in the time spent attacking the intruder male by the nNOS $-/-$ females relative to $\mathrm{WT}$ mice became apparent. In contrast, the average times spent sniffing the intruder male and in retrieving pups was statistically equivalent for the $\mathrm{nNOS}-/-$ and WT mice. One drawback of knock-out studies is that the deletion of a gene may have numerous developmental effects that are separable from the functional use of the protein product as adults (Nelson, 1997). This criticism is especially valid for the $\mathrm{nNOS}-/-$ mice given recent work showing the developmental role of NO in establishing synaptic connections in Drosophila (Gibbs and Truman, 1998). Consequently, future experiments using the pharmacological inhibition of nNOS in conjunction with maternal aggression tests will be important in determining whether the functional loss of nNOS in the adult produces the same phenotype as the nNOS $-/-$ mice.

An unexpected finding was the significantly elevated body weight of the pups of the nNOS $-/-$ mice. It is possible that the nNOS $-/-$ mothers spend more time in the nursing position relative to $\mathrm{WT}$, that the $\mathrm{nNOS}-/-$ pups show greater feeding activity than the WT pups, or both. Long-term videotaping of the 


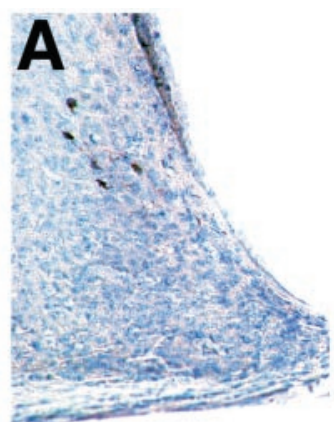

\section{Virgin female (no stimulus)}

Table 1. Numbers of cells with citrulline-IR within subregions of the hypothalamus across groups and treatments

Specific hypothalamic regions

\begin{tabular}{lccc} 
& \multicolumn{2}{l}{ Specific hypothalamic regions } \\
\cline { 2 - 4 } & MPOA & SCN & SPa \\
\hline Group (treatment) & & & \\
Lact. fem. (no stimulus) & $10.2 \pm 0.7(\mathrm{a})$ & $23.8 \pm 6.2(\mathrm{a})$ & $3.8 \pm 2.3$ \\
Lact. fem. (+ male intruder) & $27.2 \pm 3.0(\mathrm{~A})^{* * *}$ & $68.5 \pm 8.6(\mathrm{~A})^{* * *}$ & $11.1 \pm 1.3(\mathrm{~A})^{* * *}$ \\
Lact. fem (+ anesth. male intruder) & $10.0 \pm 0.4^{* * *}$ & $38.4 \pm 5.4^{*}$ & $4.2 \pm 1.0^{* *}$ \\
Virg. fem. (no stimulus) & $6.3 \pm 0.9(\mathrm{a})$ & $19.6 \pm 1.5(\mathrm{a})$ & $0.8 \pm 0.1$ \\
Virg. fem. (+ male intruder) & $11.4 \pm 1.6(\mathrm{~B})$ & $37.6 \pm 5.6(\mathrm{~B})^{*}$ & $3.8 \pm 1.0(\mathrm{~B})$ \\
Male (no stimulus) & $1.1 \pm 0.5(\mathrm{~b})$ & $1.7 \pm 0.9(\mathrm{~b})$ & $0.7 \pm 0.4$ \\
Male (+ male intruder) & $2.2 \pm 1.3(\mathrm{C})$ & $8.6 \pm 2.9(\mathrm{C})$ & $1.8 \pm 0.8(\mathrm{~B})$
\end{tabular}

Different lowercase letters (a, b, c) indicate significant differences within "no stimulus" treatment $(p<0.05)$. Different capital letters (A, B, C) indicate significant differences within "+ male intruder" treatment $(p<0.05)$. Significant differences within each group between "no stimulus" and "+ male intruder" treatments: ${ }^{*} p<0.05 ;{ }^{* *} p<0.01$; ${ }^{* * *} p<0.001$; two-way ANOVA. Significant differences between "Lact. fem. (+ anesth. male intruder)" and "Lact. fem. (+ male intruder): * $p<$ $0.05 ;{ }^{* *} p<0.01 ;{ }^{* * *} p<0.001$; unpaired Student's $t$ test.

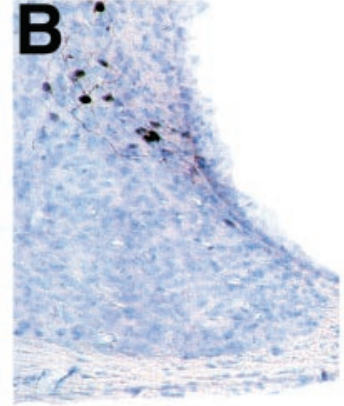

\section{Virgin female (+ male intruder)}

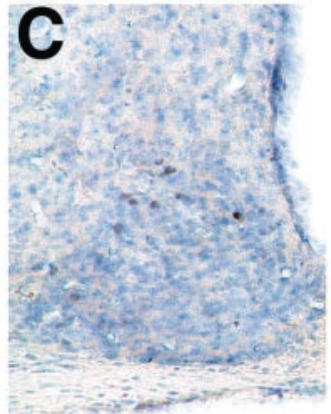

Male (+ male intruder)

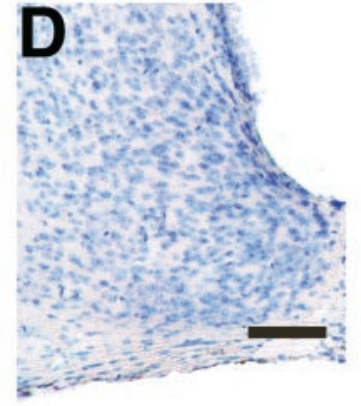

\section{Lactating nNOS-/- female (+ male intruder)}
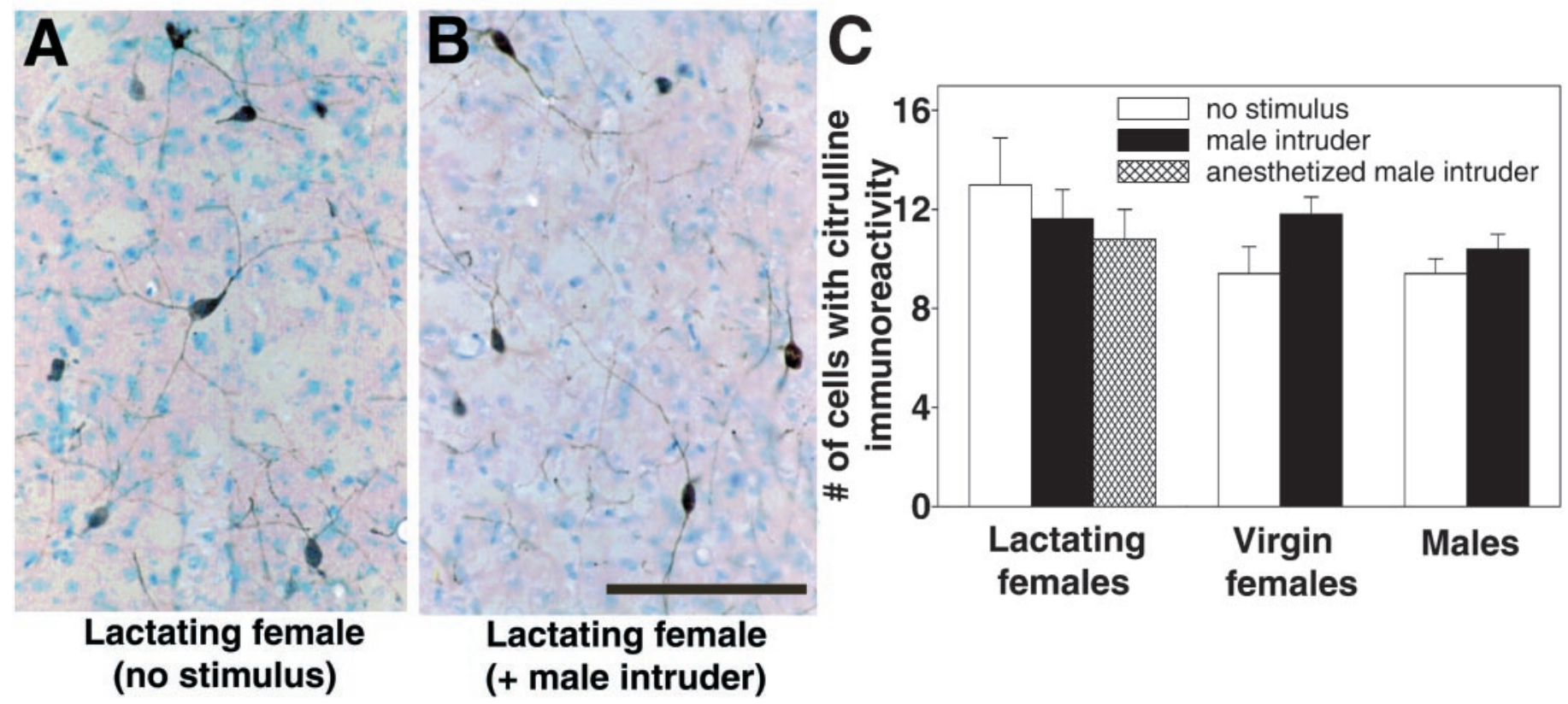

Figure 5. Top. Representative photomicrographs of citrulline-IR in unstimulated virgin females $(A)$, virgin females exposed to a male $(B)$, males exposed to a male intruder $(C)$, and nNOS-/- lactating females exposed to a male $(D)$. All sections were counterstained with thionin. Scale bar, $100 \mu \mathrm{m}$. Figure 6. Bottom. Citrulline-IR is not altered across groups or treatments in the caudate putamen region of the brain. Representative photomicrographs showing citrulline-IR in the caudate putamen of unstimulated lactating females $(A)$ and lactating females exposed to a male intruder for 10 min $(B)$. $C$, The average number of cells with citrulline-IR in the caudate putamen is almost identical across groups and treatment. Error bars represent means \pm SE. Scale bar, $100 \mu \mathrm{m}$. 
interactions of the nNOS $-/-$ females with their pups may be required to explore either of these possibilities.

\section{Relationship of citrulline-IR to NO activity}

Our study is the first to combine behavioral testing with citrulline immunohistochemistry to provide an indirect "snapshot" of NO release during a specific behavior. Thus, it is possible that anticitrulline antibodies can be used in conjunction with other behavioral tests to determine indirectly which specific cells, if any, release NO in association with those behaviors. Citrulline is the breakdown product when NO is cleaved enzymatically from arginine by NOS and can be analyzed chemically and immunohistochemically as an indirect measurement of NO production (Eliasson et al., 1997; Moroz et al., 1999). Although NO can be measured using NO-specific probes (Luo et al., 1993; Clough et al., 1998; Loeb et al., 1998), a shortcoming of these studies is that it is not always clear which specific cells are producing NO. The advantage of citrulline immunohistochemistry is that specific cells producing NO can be identified. Although citrulline is transiently produced as part of the urea cycle, previous work indicates that citrulline immunohistochemistry can be a reliable, indirect indicator of NO release in the mouse CNS. First, citrulline-IR was only found to be present in neurons that also contained NOS (Eliasson et al., 1997). Second, the use of NOS inhibitors and the deletion of the nNOS gene eliminated citrulline-IR from the mouse brain (Eliasson et al., 1997). Third, no detectable levels of the mRNA of an important urea cycle enzyme were detected in the mouse brain (Eliasson et al., 1997). Furthermore, in mammals, the degradation of amino acids in the urea cycle occurs almost exclusively in the liver. Thus, it is likely that the increase in the number of citrulline-positive cells that are exhibited in association with maternal aggression reflect an increase in NO production. Nonetheless, in future work it will be useful to use NO-specific probes during the production of a behavior followed by citrulline-IR to properly quantify and confirm NO production.

\section{Possible interpretations of increased numbers of citrulline-positive cells during maternal aggression}

This study indicates that an increase in citrulline-IR in the MPOA, SCN, and SPa regions of the hypothalamus, but not in other regions of the brain (Figs. 3, 4, 6), is associated with maternal aggression. These results indicate, indirectly, that NO release is associated with maternal aggression. Whether, this increased synthesis of citrulline (and NO) is directly, indirectly, or not at all related to maternal aggression is unknown. Evidence from previous studies links the citrulline-positive areas of the hypothalamus to the production of maternal aggression. Citrulline-positive cells of the MPOA and SPa lie in and just dorsal to the peri-SCN region (Fig. 3). In the cat, electrical stimulation of the hypothalamus just dorsal to the SCN and just lateral to the third ventricle elicits defensive rage, which is thought to be equivalent to maternal aggression (Siegel et al., 1999). Through lesion studies in the rat, the paraventricular nucleus and the ventromedial hypothalamus have been implicated in the control of maternal aggression (Hansen, 1989; Giovenardi et al., 1998). The MPOA has been implicated in some maternal behaviors relating to the care of the pups but not maternal aggression per se (Numan, 1990; Pedersen et al., 1994). Unfortunately, site-directed studies of maternal aggression have not been performed in mice. Consequently, the work in cat provides the most suggestive evidence to date that there may be a link between the MPOA, SCN, and SPa regions of the hypothalamus and maternal aggression.
Although the SCN is best understood for its role in the control of circadian function (Stephan and Zucker, 1972), the levels of AVP in the SCN have been positively correlated with nestbuilding behavior in mice (Bult et al., 1992). Also, some AVPpositive cells in the mouse SCN project to other hypothalamic areas, such as the paraventricular nucleus, in which they could influence noncircadian pathways, including maternal aggression (Vrang et al., 1995). Based on preliminary examinations, it appears that some of the cells exhibiting citrulline-IR during maternal aggression also contain AVP (our unpublished observations). Because NO can act as an intracellular signal, an intercellular signal, or both (Holscher, 1997; Park et al., 1998), and because of the excitatory role AVP plays in male aggression, it will be important to examine the possible link between citrulline-IR, NO release, and AVP release during maternal aggression.

To help determine the significance of the elevation in citrulline, it will be useful to identify both the cells releasing NO and those responding to it in the hypothalamus. In rats, oxytocin, a peptide linked to maternal aggression (Giovenardi et al., 1997), and nNOS are coexpressed in some cells of the MPOA (Yamada et al., 1996), but double-labeling experiments using citrulline and neuropeptides, or neurotransmitters, will be required to identify specifically which cells are producing NO in aggressive lactating female mice. The most common pathway for NO signaling is the activation of soluble guanylate cyclases, and future studies using cGMP immunohistochemical and double-labeling techniques will also be of great help in identifying possible targets of NO in the MPOA, SCN, and SPa regions of the hypothalamus.

An important question is why the virgin and lactating females differ in terms of behavior and brain histology when confronted with the same stimuli of the intruder male. From a mechanistic level, one possibility is that the lactating females express increased levels of nNOS in the brain relative to virgin females. Indeed, steroid hormones associated with pregnancy and lactation have been shown to increase NOS synthesis in the hypothalamus (Ceccatelli and Eriksson, 1993; Ceccatelli et al., 1996; Luckman et al., 1997; Popeski et al., 1999). If nNOS is upregulated in the MPOA, SCN, and SPa regions of the hypothalamus during pregnancy and lactation, then this could explain why the unstimulated virgin females exhibit lower levels of citrulline-IR than the unstimulated lactating females. Also, it could explain why the same sensory stimuli (the male intruder) produced significantly fewer citrulline-positive cells in the virgins as in the lactating females.

The issue of changes in nNOS expression is currently being explored, but the question remains whether the differences in citrulline-IR somehow reflect the underlying basis for the difference in behavior between the virgin and lactating females. At this stage, we have no direct evidence that differences in NO production in lactating females underlie maternal aggression. Given the statistically significant positive correlation found between the number of citrulline-positive cells and time spent attacking an intruder, it is tempting to speculate that indeed NO is directly, or indirectly, involved in maternal aggression. Furthermore, the females exposed to the immobile intruder males had the almost identical sensory stimuli as those presented with the active male, but still they lacked the increases in citrulline-IR and, perhaps significantly, they did not exhibit maternal aggression. Nonetheless, without direct evidence, any role for NO in maternal aggression is still speculation.

For the virgin and the lactating female, the presence of an 
intruder male can present different implications. In the first case, a male could pose as a potential threat to the pups of the lactating female but pose as a potential mate for the virgin female. During our testing times (8:00 A.M. to 12:00 P.M.), however, females are not normally in proestrus and in no cases did we see any lordosis behavior by any of the females. Also, except for the first day after birth, lactating females are not normally sexually receptive (Svare, 1990). In male rats, however, NO production in the MPOA does positively correlate with sexual behavior (Sato et al., 1998). If increases in citrulline-IR in females do relate to sexual behavior, then it would need to be determined why lactating females exhibit significantly higher numbers of citrulline-positive cells than virgins. Another consideration is that NO can also have inhibitory actions on its target cells. Thus, the change in citrulline (or NO) synthesis by the presence of the male could reflect the inhibition, and not the activation, of certain neural pathways and behaviors.

The possibility that high levels of citrulline synthesis are associated with all forms of aggression was ruled out by the studies on male mice. The numbers of citrulline-positive cells of aggressive and nonaggressive males were equivalent but were significantly lower than for any of the female groups or treatments (Fig. 4). If males tonically release NO to inhibit aggression, then one would expect a decrease in citrulline (and NO) synthesis during an aggressive test. This presumed decrease in citrulline may be difficult to detect immunohistochemically if the half-life of citrulline is longer than the $10 \mathrm{~min}$ test period. That the pattern of citrulline-IR in the hypothalamus is sexually dimorphic indicates, indirectly, that the pattern of NO release in females underlies a behavior or physiology that is specific to females.

Because the elimination of nNOS activity in males increases aggression (Nelson et al., 1995; Demas et al., 1997), suggesting that NO inhibits male aggression, it would be interesting if females use NO to activate aggression. A specific implication of our work is that the synthesis of citrulline (and, indirectly, NO) increases dramatically within a discrete group of cells in association with maternal aggression. Whether, or how, the actions of these cells contribute to maternal aggression is currently being determined. A general implication of our work is that behavioral testing and citrulline immunohistochemistry can be successfully combined as a technique to gain valuable, indirect information about the specific site of NO release during a behavior. This technique, then, provides a new tool for the study of neural circuits and behavior.

\section{REFERENCES}

Bredt DS, Snyder SH (1992) Nitric oxide, a novel neuronal messenger. Neuron 8:3-11.

Bridges RS (1996) Biochemical basis of parental behavior in the rat. In: Parental care: evolution, mechanisms, and adaptive significance (Rosenblatt JS, Snowden CT, eds), pp 215-237. San Diego: Academic.

Bult A, van der Zee EA, Compaan JC, Lynch CB (1992) Differences in the number of arginine-vasopressin-immunoreactive neurons exist in the suprachiasmatic nuclei of house mice selected for differences in nest-building behavior. Brain Res 578:335-338.

Ceccatelli S, Eriksson M (1993) The effect of lactation on nitric oxide synthase gene expression. Brain Res 625:177-179.

Ceccatelli S, Grandison L, Scott RE, Pfaff DW, Kow LM (1996) Estradiol regulation of nitric oxide synthase mRNAs in rat hypothalamus. Neuroendocrinology 64:357-363.

Clough GF, Bennett AR, Church MK (1998) Measurement of nitric oxide concentration in human skin in vivo using dermal microdialysis. Exp Physiol 83:431-434.
Demas GE, Eliasson MJ, Dawson TM, Dawson VL, Kriegsfeld LJ, Nelson RJ, Snyder SH (1997) Inhibition of neuronal nitric oxide synthase increases aggressive behavior in mice. Mol Med 3:610-616.

Eliasson MJ, Blackshaw S, Schell MJ, Snyder SH (1997) Neuronal nitric oxide synthase alternatively spliced forms: prominent functional localizations in the brain. Proc Natl Acad Sci USA 94:3396-3401.

Ferris CF, Melloni Jr RH, Koppel G, Perry KW, Fuller RW, Delville Y (1997) Vasopressin/serotonin interactions in the anterior hypothalamus control aggressive behavior in golden hamsters. J Neurosci 17:4331-4340.

Franklin KBJ, Paxinos G (1997) The mouse brain in stereotaxic coordinates. San Diego: Academic.

Gibbs SM, Truman JW (1998) Nitric oxide and cyclic GMP regulate retinal patterning in the optic lobe of Drosophila. Neuron 20:83-93.

Giovenardi M, Padoin MJ, Cadore LP, Lucion AB (1997) Hypothalamic paraventricular nucleus, oxytocin, and maternal aggression in rats. Ann NY Acad Sci 807:606-609.

Giovenardi M, Padoin MJ, Cadore LP, Lucion AB (1998) Hypothalamic paraventricular nucleus modulates maternal aggression in rats: effects of ibotenic acid lesion and oxytocin antisense. Physiol Behav 63:351-359.

Hansen S (1989) Medial hypothalamic involvement in maternal aggression of rats. Behav Neurosci 103:1035-1046.

Holscher C (1997) Nitric oxide, the enigmatic neuronal messenger: its role in synaptic plasticity. Trends Neurosci 20:298-303.

Huang PL, Dawson TM, Bredt DS, Snyder SH, Fishman MC (1993) Targeted disruption of the neuronal nitric oxide synthase gene. Cell 75:1273-1286.

Kendrick KM, Guevara-Guzman R, Zorrilla J, Hinton MR, Broad KD, Mimmack M, Ohkura S (1997) Formation of olfactory memories mediated by nitric oxide. Nature 388:670-674.

Kordon C, Blake CA, Terkel J, Sawyer CH (1973) Participation of serotonin-containing neurons in the suckling-induced rise in plasma prolactin levels in lactating rats. Neuroendocrinology 13:213-223.

Loeb AL, Raj NR, Longnecker DE (1998) Cerebellar nitric oxide is increased during isoflurane anesthesia compared to halothane anesthesia: a microdialysis study in rats. Anesthesiology 89:723-730.

Luckman SM, Huckett L, Bicknell RJ, Voisin DL, Herbison AE (1997) Up-regulation of nitric oxide synthase messenger RNA in an integrated forebrain circuit involved in oxytocin secretion. Neuroscience 77:37-48.

Luo D, Knezevich S, Vincent SR (1993) $N$-methyl-D-aspartate-induced nitric oxide release: an in vivo microdialysis study. Neuroscience 57:897-900.

Maestripieri D (1990) Maternal aggression and litter size in the female house mouse. Ethology 84:27-34.

Mann MA, Konen C, Svare B (1984) The role of progesterone in pregnancy-induced aggression in mice. Horm Behav 18:140-160.

Moroz LL, Gillette R, Sweedler JV (1999) Single-cell analyses of nitrergic neurons in simple nervous systems. J Exp Biol 202:333-341.

Nelson RJ (1997) The use of genetic "knockout" mice in behavioral endocrinology research. Horm Behav 31:188-196.

Nelson RJ, Demas GE, Huang PL, Fishman MC, Dawson VL, Dawson TM, Snyder SH (1995) Behavioural abnormalities in male mice lacking neuronal nitric oxide synthase. Nature 378:383-386.

Numan M (1990) Neural control of maternal behavior. In: Mammalian parenting: biochemical, neurobiological, and behavioral determinants (Krasnegor NA, Bridges RS, eds), pp 231-259. New York: Oxford UP.

Okere CO, Higuchi T, Kaba H, Russell JA, Okutani F, Takahashi S, Murata T (1996) Nitric oxide prolongs parturition and inhibits maternal behavior in rats. NeuroReport 7:1695-1699.

Olivier B, Mos J, van Oorschot R, Hen R (1995) Serotonin receptors and animal models of aggressive behavior. Pharmacopsychiatry [Suppl] 2:80-90.

Park JH, Straub VA, O'Shea M (1998) Anterograde signaling by nitric oxide: characterization and in vitro reconstitution of an identified nitrergic synapse. J Neurosci 18:5463-5476.

Parmigiani S, Ferrari PF, Palanza P (1998) An evolutionary approach to behavioral pharmacology: using drugs to understand proximate and ultimate mechanisms of different forms of aggression in mice. Neurosci Biobehav Rev 23:143-153.

Pasqualotto BA, Hope BT, Vincent SR (1991) Citrulline in the rat brain: immunohistochemistry and coexistence with NADPH-diaphorase. Neurosci Lett 128:155-160. 
Pedersen CA, Caldwell JD, Walker C, Ayers G, Mason GA (1994) Oxytocin activates the postpartum onset of rat maternal behavior in the ventral tegmental and medial preoptic areas. Behav Neurosci 108:1163-1171.

Popeski N, Amir S, Woodside B (1999) Changes in NADPH-d staining in the paraventricular and supraoptic nuclei during pregnancy and lactation in rats: role of ovarian steroids and oxytocin. J Neuroendocrinol 11:53-61.

Sato Y, Horita H, Kurohata T, Adachi H, Tsukamoto T (1998) Effect of the nitric oxide level in the medial preoptic area on male copulatory behavior in rats. Am J Physiol 274:R243-R247.

Siegel A, Roeling TA, Gregg TR, Kruk MR (1999) Neuropharmacology of brain-stimulation-evoked aggression. Neurosci Biobehav Rev 23:359-389.

Stephan FK, Zucker I (1972) Circadian rhythms in drinking behavior and locomotor activity of rats are eliminated by hypothalamic lesions. Proc Natl Acad Sci USA 69:1583-1586.

Stern JM, Kolunie JM (1993) Maternal aggression of rats is impaired by cutaneous anesthesia of the ventral trunk, but not by nipple removal. Physiol Behav 54:861-868.

Stern JM, McDonald C (1989) Ovarian hormone-induced short-latency maternal behavior in ovariectomized virgin Long-Evans rats. Horm Behav 23:157-172.

Svare B (1990) Maternal aggression: hormonal, genetic, and developmental determinants. In: Mammalian parenting: biochemical, neurobiological, and behavioral determinants (Krasnegor NA, Bridges RS, eds), pp 118-132. New York: Oxford UP.

Svare B, Betteridge C, Katz D, Samuels O (1981) Some situational and experiential determinants of maternal aggression in mice. Physiol Behav 26:253-258.

Thomas SA, Palmiter RD (1997) Impaired maternal behavior in mice lacking norepinephrine and epinephrine. Cell 91:583-592.

Vrang N, Larsen PJ, Mikkelsen JD (1995) Direct projection from the suprachiasmatic nucleus to hypophysiotrophic corticotropin-releasing factor immunoreactive cells in the paraventricular nucleus of the hypothalamus demonstrated by means of Phaseolus vulgarisleucoagglutinin tract tracing. Brain Res 684:61-69.

Wolff JO (1985) Maternal aggression as a deterrent to infanticide in Peromyscus leucopus and P. maniculatus. Anim Behav 33:117-123.

Yamada K, Emson P, Hokfelt T (1996) Immunohistochemical mapping of nitric oxide synthase in the rat hypothalamus and colocalization with neuropeptides. J Chem Neuroanat 10:295-316. 